\title{
Transition region explosive events: Do they have a coronal counterpart?
}

\author{
L. Teriaca ${ }^{1}$, M. S. Madjarska² ${ }^{2}$ and J. G. Doyle ${ }^{2}$ \\ 1 Osservatorio Astrofisico di Arcetri, 50125 Firenze, Italy \\ 2 Armagh Observatory, College Hill, Armagh BT61 9DG, N. Ireland
}

Received 22 February 2002 / Accepted 27 May 2002

\begin{abstract}
EUV explosive events are a prominent class of phenomena characterizing the solar transition region. Their correct location in the wider frame-work of the outer solar atmosphere can provide important insight on the nature of the transition region itself and its relationship with the hotter corona and the cooler chromosphere. In this paper we present new high-cadence SUMER observations of the "quiet" Sun obtained simultaneously in the mid-transition region N v $1238.8 \AA$ line $\left(1.8 \times 10^{5} \mathrm{~K}\right)$ and in the coronal $\mathrm{Mg}$ x $625 \AA$ line $\left(1.1 \times 10^{6} \mathrm{~K}\right)$. These observations are aimed at providing information on the behaviour of the coronal plasma during EUV transition region explosive events detected in N v $1238.8 \AA$. None of the events observed in N v shows any detectable signature in the $\mathrm{Mg}$ x line profile or in its integrated intensity. The analysis of 1996 observations obtained simultaneously in N v $1238.8 \AA$ and $\mathrm{S}_{\text {II }} 1253.8 \AA\left(3.5 \times 10^{4} \mathrm{~K}\right)$ shows, instead, a weak but clear presence of enhanced wings in the $\mathrm{S}$ II line profile during a series of events observed in $\mathrm{N}$ v. These results suggest that EUV explosive events are not directly relevant in heating the corona and are characteristic of structures not obviously connected with the upper corona. The evidence of a chromospheric response suggests that, contrary to some previous suggestions, explosive events have a chromospheric origin.
\end{abstract}

Key words. Sun: transition region, explosive events, corona - UV radiation

\section{Introduction}

When observed with high spatial $(\sim 1$ arcsec) and temporal $(\sim 10-100 \mathrm{~s})$ resolution the "quiet" Sun transition region is anything but "quiet" showing, together with quasi-periodic fluctuations of the intensity and shift of ultraviolet emission lines (O'Shea et al. 2001; Banerjee et al. 2001) also transient events such as brightening (Doyle et al. 1998; Gallagher et al. 1999; Brković et al. 2000 and references therein), blinkers (strong transient brightenings on time-scales of tens of minutes; Harrison 1997; Harrison et al. 1999) and UV explosive events and jets (Brueckner \& Bartoe 1983). In particular, UV explosive events are a class of dynamic events quite common in "quiet" Sun areas, with a birthrate over the whole Sun of between $600 \mathrm{~s}^{-1}$ (Dere et al. 1989) and $3300 \mathrm{~s}^{-1}$ (Ryutova \& Tarbell 2000). A similar birthrate is observed also on disk areas within coronal holes (Dere 1992). Explosive events are characterized by spatial scales of $\sim 2000 \mathrm{~km}$, average lifetime of about $60 \mathrm{~s}$ and highly non-Gaussian line profiles showing Doppler shifts up to $250 \mathrm{~km} \mathrm{~s}^{-1}$ (Dere et al. 1989) and often appear in bursts lasting up to $30 \mathrm{~min}$ (Innes et al. 1997a; Chae et al. 1998a; Pérez et al. 1999). They are generally

Send offprint requests to: L. Teriaca,

e-mail: 1te@arcetri.astro.it

or http://star.arm.ac.uk/preprints/ observed at the boundaries of the super-granulation cells (Porter \& Dere 1991) in regions with weak and mixed polarity fluxes away from the brightest network regions (Chae et al. 1998a). Some of them have also been observed in dark cellcenter areas (Wilhelm et al. 1998). The often observed association with episodes of photospheric magnetic flux cancellation (Dere et al. 1991; Chae et al. 1998a; Ryutova \& Tarbell 2000) indicates magnetic reconnection to be their likely energy source.

Despite the fact that UV explosive events have been observed for almost 20 years (Brueckner \& Bartoe 1983; Dere et al. 1989; Dere et al. 1991; Porter \& Dere 1991; Dere 1994; Innes 1997a, 1997b; Wilhelm et al. 1998; Chae et al. 1998a, 1998b; Pérez et al. 1999; Landi et al. 2000; Ryutova \& Tarbell 2000; Teriaca et al. 2001) their location in the wider framework of the outer solar atmosphere is still uncertain since the large majority of the observational work was restricted to lines formed below $10^{6} \mathrm{~K}$. Dere (1992), from the analysis of HRTS spectra, reports that less than $1 \%$ of the explosive events observed in transition region lines are also seen in chromospheric lines such as $C_{\text {I }} 1561 \AA$, while they are weakly seen in C II $1335 \AA$. However, taking advantage of the higher sensitivity of the SUMER spectrograph, Madjarska \& Doyle (2002) offered further evidence that transition region explosive events appear in chromospheric lines such as $\mathrm{O}$ I and the optically 
thick low temperature line Ly 6. Wilhelm et al. (1998) show that explosive events clearly appear in all lines formed between $10^{5} \mathrm{~K}^{1}(\mathrm{~N}$ III $763 \& 764 \AA)$ and $2.5 \times 10^{5} \mathrm{~K}(\mathrm{O}$ v multiplet around $760 \AA$ ) as well as, in the case of two events within active regions, in Ne vIII $770 \AA\left(6.3 \times 10^{5} \mathrm{~K}\right)$ and $\mathrm{Mg}$ IX $749 \AA$ $\left(9.8 \times 10^{5} \mathrm{~K}\right)$. A third event, observed in the dark cell center was not seen, instead, in lines formed above $2.5 \times 10^{5} \mathrm{~K}$.

The results of an attempt to observe the coronal counterparts to explosive events were presented by Dere (1992) and Moses \& Cook (1994). Observations were carried out during the near simultaneous sounding rocket flights of the NRL UV spectrograph (HRTS-5) and the AS\&E X-ray telescope on December 1987. In general, no direct coronal counterpart to the explosive events observed in C Iv by HRTS was detected. Transition region explosive events did not correspond to coronal X-ray Bright Points (XBPs), however, a correlation was observed between the explosive events and the overlying largescale coronal structure. The number density of C IV events was twice as large inside as outside a dark coronal lane as seen by the X-ray imager (Moses \& Cook 1994). Dynamic Coronal High Velocity Events (CHVEs) very similar to the transition region explosive events, but with velocities on the order of 200$500 \mathrm{~km} \mathrm{~s}^{-1}$, have been detected in coronal and upper transition region lines observed with the NRL slitless spectroheliograph S082A on Skylab (Brueckner et al. 1976; Cheng \& KjeldsethMoe 1990). Most of these events occur in active regions although some occur in quiet regions but their maximum temperatures is less than $\sim 10^{6} \mathrm{~K}$. Although the S082A spectroheliograms cover the entire solar disk, much more transition region explosive events than CHVEs were observed. Since explosive events are not particularly bright, Kjeldseth-Moe \& Cheng (1994) have suggested that it may be impossible to detect most of their upper transition region and coronal counterparts in the heliographic images.

Koutchmy et al. (1997) provided evidence, through Yohkoh-SXT observations, for the existence of a new type of soft X-ray (SXR) brightening events characterized by timescales of about $90 \mathrm{~s}$, spatial scales from a few to several arc-seconds and energies of $\sim 10^{24}$ erg (just above the detection threshold) or higher. These events, named by the authors coronal flashes, were observed against the dark background of coronal holes near the solar activity minimum with an occurrence rate $\sim 200$ times smaller than that of explosive events. However, also in this case, the majority of the events could be lost below the detection threshold. Coronal flashes could represent the low energy tail of the SXR events observed with Yohkoh-SXT above the quiet Sun network by Krucker et al. (1997). Finally, Innes (2001) reports some explosive events to be associated with regions of enhanced emission observed with TRACE in the $171 \AA$ wave-band (centered around $173 \AA$ ), including strong coronal lines such as Fe IX 171.07 and Fe x $174.53 \AA$.

In an effort to resolve the coronal contribution of explosive events we present new observations of the quiet Sun

\footnotetext{
1 The line formation temperatures used throughout this paper are obtained using the data tables of the Arcetri Spectral Code (Landi \& Landini 1998).
}

transition region and corona obtained in the $\mathrm{N} v 1238.8 \AA$ and $\mathrm{Mg}$ x $625 \AA$ (observed in second order at $1250 \AA$ ) lines recorded simultaneously with the SUMER spectrograph. These observations, avoiding the difficulties of comparing observations obtained with instruments characterized by different resolutions, sensitivity and spectral responses, are able to determine whether or not explosive events detected in $\mathrm{N} v$ have an observable counterpart in the $1.1 \mathrm{MK}$ coronal plasma in which the $\mathrm{Mg} \mathrm{x}$ line is formed, providing an important constrain to theoretical works on transition region structuring and coronal heating. In addition, we also look at $\mathrm{N} v$ and $\mathrm{S}$ II data in order to quantify the chromospheric versus transition region behaviour during explosive events.

\section{Observations and data reduction}

The characteristics and capabilities of the SUMER spectrograph aboard SoHO are thoroughly discussed by Wilhelm et al. (1995, 1997) and Lemaire et al. (1997). Here we only report some characteristics of the SUMER detectors that are particularly relevant to the present work. SUMER is equipped with two photon-counting detectors (A and B) operating in Cross Delay Line (XDL) technique (for details see Siegmund et al. 1994). The central area of the SUMER detectors is coated with a potassium-bromide $(\mathrm{KBr})$ layer which increases the detective quantum efficiency up to a factor $\sim 10$ in the range from $900 \AA$ to $1600 \AA$. The rotation of a flat mirror allows coverage of the spectral range 780-1610 $\AA$ with detector A (660$1500 \AA$ with detector $\mathrm{B}$ ), thus recording also second order lines between 390 and $805 \AA$ (330 and $750 \AA$ with detector B). However, the fall-off in the reflectivity of the siliconcarbide (SiC) mirrors severely reduces the possibility to observe lines at wavelength below $500 \AA$ A Second order lines (500-800 ^) are largely insensitive to the KBr coating, allowing us to recognize them from the first order ones using the different wavelength-dependent sensitivity of $\mathrm{KBr}$ compared to the bare part. In the case of detector A (the one used to obtain all the observations discussed in this work) the sensitivity ratio between the $\mathrm{KBr}$ and the bare detector is 10.23 for first order wavelengths around $1250 \AA$ compared to $\sim 1.5$ for second order ones.

\subsection{First order contribution}

The Mg x $625 \AA$ line is observed by SUMER as a second order line around $1250 \AA$. It, hence, overlaps with several chromospheric and low transition region first order lines from ions such as C I, S II and Si II (see Fig. 1). Madjarska \& Doyle (2002) have shown that during large explosive events the spectral radiances at line peak of optically thin $\mathrm{C}_{\mathrm{I}}$ and $\mathrm{O}_{\mathrm{I}}$ lines increase of a factor between 1.6 and 2. In order to establish whether the observed variation (if any) in the $\mathrm{Mg} \mathrm{x}$ line profile and/or in its integrated intensity are not induced by the variation of the chromospheric spectrum, it is necessary to make sure that contributions coming from overlapping first order lines and background are negligible.

Using the NRL spectrograph S082B on Skylab-ATM, Cohen et al. (1978) report the presence of two lines at 1249.81 


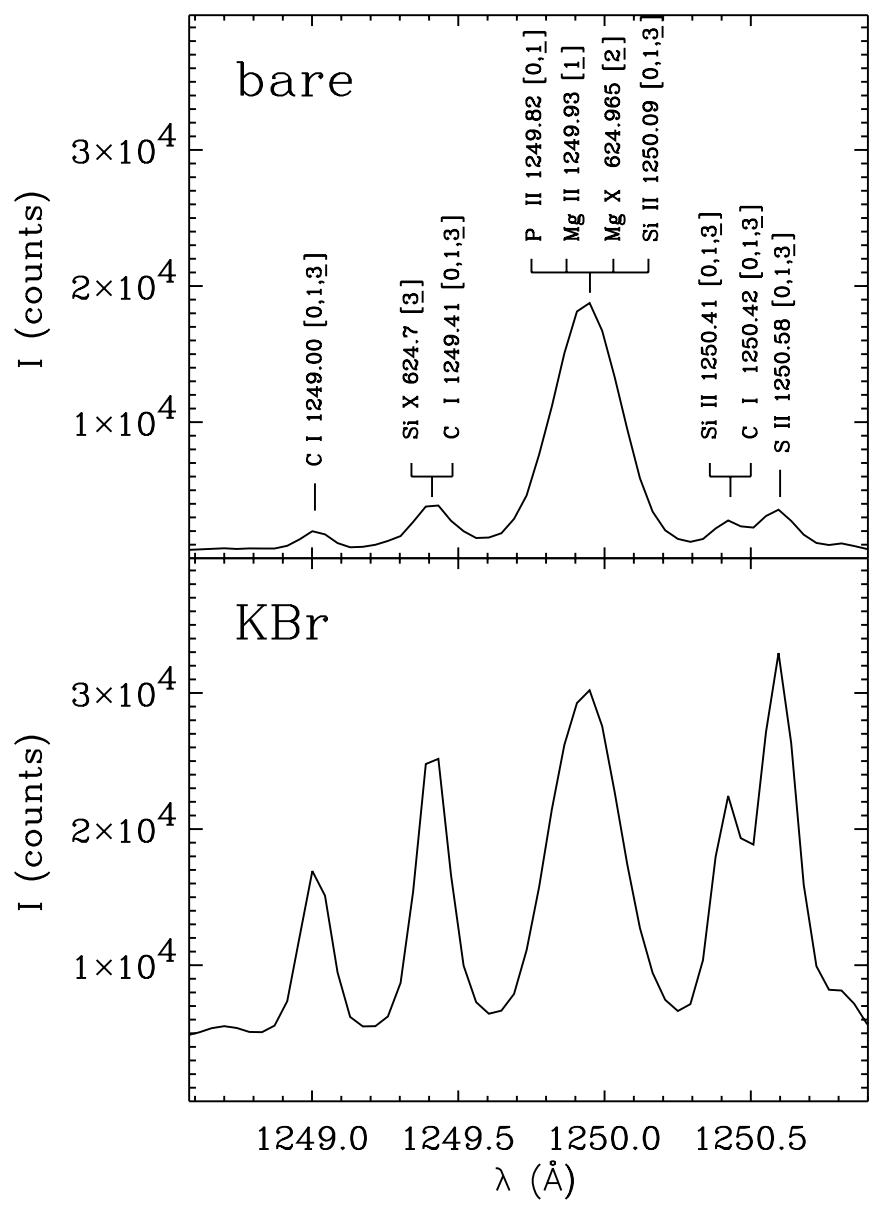

Fig. 1. 1996 January 30 average quiet Sun SUMER spectra around $1250 \AA$ recorded on the $\mathrm{KBr}$-coated (bottom panel) and on the bare (top panel) parts of detector A, respectively. It is evident the tenfold reduction of the bare detector sensitivity at first order wavelengths with respect to the $\mathrm{KBr}$-coated detector. References: Cohen et al. (1978) [0]; Sandlin et al. (1986) [1]; Dammasch et al. (1999) [2]; Curdt et al. (2001) [3]. Underlined references indicate from where the displayed observed wavelengths have been taken.

and $1250.09 \AA$ during a flare. These two features are identified as P II $1249.82 \AA$ and Si II $1250.089 \AA$ (see Fig. 1). Both lines are reported to have spectral radiance equal to 0.5 that of $C_{\text {I }} 1249.004$. We use C I 1249.004 as a reference since it is not affected by blending. The presence of these two lines is confirmed by Sandlin et al. (1986) who observe them at 1249.812 and $1250.089 \AA$, respectively. These authors find in a plage, using the HRTS NRL spectrograph, spectral radiances at line peak above the background of 0.4 and 0.9 that at the peak of $\mathrm{C}_{\text {I }} 1249.004 \AA$ for P II and Si II, respectively. They also observe a third spectral line at $1249.932 \AA$ showing a peak spectral radiance above the background of 0.37 that at the peak of C I 1249.004. Sandlin et al. (1986) identify this line as Mg II 1249.932. Assuming, as a first approximation, all these spectral lines to have the same line width, the above relative amplitudes translate to relative spectral radiances.

Finally, Curdt et al. (2001) report the presence of the Si II $1250.089 \AA$ line and give a peak spectral radiance of 0.65 that at the peak of C I $1249.004 \AA$ (background included) in

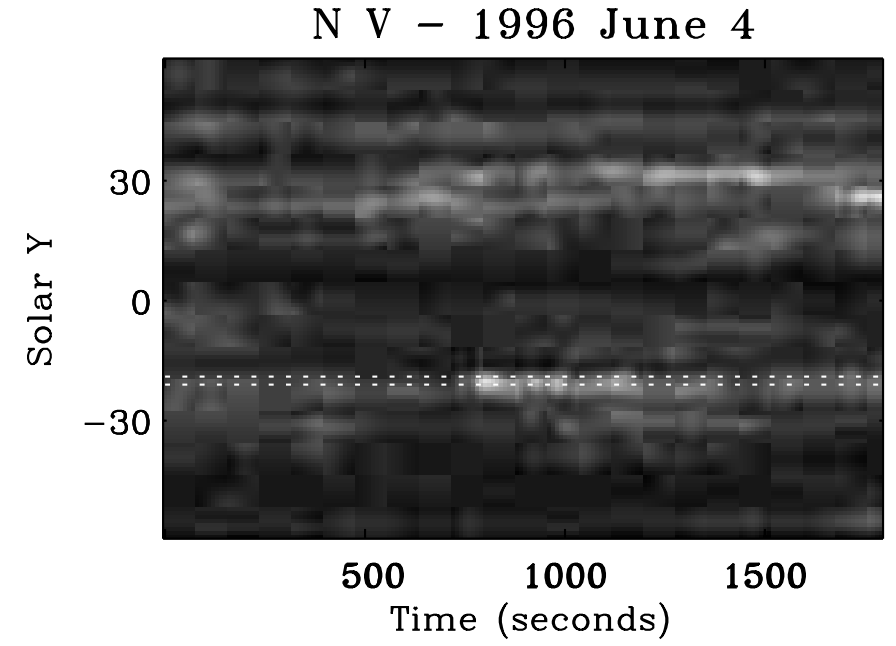

Fig. 2. N v 1238.8 intensity map of the 1996 June 4 dataset. The horizontal dashed lines define the portion of the slit along which a large explosive event was observed around $T \sim 800 \mathrm{~s}$.

a quiet Sun area. In Fig. 1 is shown an example of the average quiet Sun SUMER spectrum around $1250 \AA$ recorded on the $\mathrm{KBr}$-coated (bottom panel) and bare (top panel) parts of detector A on 1996 January 30. Assuming the C I 1249.004 and $\mathrm{Si}$ II $1250.089 \AA$ spectral lines to have the same width and measuring the ratio between the $C_{\text {I }} 1249.004$ line amplitude and its background from the quiet Sun $\mathrm{KBr}$ spectrum in Fig. 1, the Curdt et al. measurement allows us to estimate the $\mathrm{Si}$ II $1250.089 \AA$ line to have a total intensity equal to 0.5 that of C I 1249.004 in quiet Sun areas.

When the second order $\mathrm{Mg} x$ line is recorded on $\mathrm{KBr}$, the first order lines show comparable line peak strengths. From the measured C I 1249.004 intensity ( 20\% that of Mg x) we can estimate that $\mathrm{Si}$ II 1250.089 accounts for $\sim 10 \%$ of the $\mathrm{Mg} \mathrm{x}$ total counts on $\mathrm{KBr}$. Another few percent may come from $\mathrm{Mg}$ II and $\mathrm{P}$ II leading to a total contribution between 10 and $15 \%$ in the case of quiet Sun observations. A visual inspection of microdensitometer reading of an HRTS spectrum (not including second order contribution) confirms our estimate. This means that the above total contribution will be of the order of $1-2 \%$ in quiet Sun areas outside explosive events when the spectrum is recorded on the bare detector. During explosive events such contributions should still be below 5\% (assuming as typical the intensity increases of chromospheric lines observed by Madjarska \& Doyle 2002). Variations above this value in the $\mathrm{Mg} \mathrm{x}$ line profile and/or integrated intensity should be safely regarded as not due to first order contributions. In any case, possible enhancements of the first order spectrum during explosive events can be assessed from C I 1249.00 and other lines still visible in the bare spectrum (see Fig. 1).

The reduction of SUMER raw images followed several stages such as local gain correction, flat-field and geometrical distortion correction. The signal to noise level is determined by the photon statistics. 


\section{2. $N \vee \& S \|$}

In order to better quantify the behaviour of the chromospheric first order spectrum during UV explosive events (detected in N v), we analyzed archive observations obtained on 1996 June 4 (hereafter DTS1) to study the temporal evolution of a quiet Sun region at Sun centre (see Fig. 2). Spectra were recorded exposing for $10 \mathrm{~s}$ the central part of detector A through the $1 \times 120 \operatorname{arcsec}^{2}$ slit. For each spectrum two spectral windows, centered at $1238.8 \AA$ (comprising N v 1238.8) and $1253.6 \AA$ (comprising S I $1253.32+C_{\text {I }} 1253.47, S_{\text {II }} 1253.8$, $\mathrm{H}_{2} 1254.11$ and $\mathrm{C}_{\mathrm{I}} 1254.51$; from Curdt et al. 2001) were transmitted to the ground. The observations discussed here started at 11:09:37 UT and consist of 180 consecutive exposures.

\section{3. $N \vee \& M g$}

High cadence simultaneous observations in N v $1238.8 \AA$ and Mg x $625 \AA$ (observed as a second order line) were planned and obtained using the SUMER spectrograph aboard SoHO. These observations were obtained during two observing campaigns on 2001 May 24 and October 23 (hereafter DTS2 and DTS3) both consisting of a sequences of spectra of a quiet region at Sun centre (see Figs. 5 and 6, respectively). In both cases the $1 \times 120 \operatorname{arcsec}^{2}$ slit centered on the bottom part of detector A was used with an exposure time of $20 \mathrm{~s}$ obtaining a temporal sequence, consisting of three spectral windows $(120$ spatial $\times 50$ spectral pixels) centered on $\mathrm{N} v 1238.8$, $\mathrm{N}$ v 1242.8 (including also the Fe xII line at $1240 \AA$ ) and $\mathrm{Mg}$ x 625 . The flat mirror was rotated in such a way to record the $\mathrm{N} v$ doublet on the $\mathrm{KBr}$-coated detector while the $\mathrm{Mg}$ x line was detected on bare.

\section{Data analysis and results}

Several explosive events were identified in these datasets examining the $\mathrm{N} v 1238$ line profiles. Here the largest event found in each dataset is discussed in detail.

\subsection{DTS1: Chromospheric and transition region behaviour}

A large explosive event (EE1) was observed in $\mathrm{N} v$ around solar $Y=-20^{\prime \prime}, \sim 800 \mathrm{~s}$ after the start of the observations. Binning over two consecutive spectra $(20 \mathrm{~s})$ and over three spatial pixels $\left(3^{\prime \prime} \simeq 2100 \mathrm{~km}\right)$ centered on the explosive event location was performed, allowing the study of the temporal behaviour of the spectrum around 1238.8 and $1253 \AA$ (see Fig. 2). In order to describe the development of enhanced wings due to explosive event activity we define, for each $\mathrm{N} v$ line profile obtained after binning, the quantity $W_{\mathrm{e}}$ (wings enhancement) defined as

$W_{\mathrm{e}}=\frac{I_{\mathrm{b}}+I_{\mathrm{r}}}{I_{\text {peak }}}$

where $I_{\text {peak }}, I_{\mathrm{b}}$ and $I_{\mathrm{r}}$ are the total counts over 3 pixels $(0.13 \AA)$ wide spectral intervals centered at line centre and 5 pixels $(0.22 \AA)$ away from the central position on the blue and on the red sides of the spectral line, respectively. In Fig. 3 (top
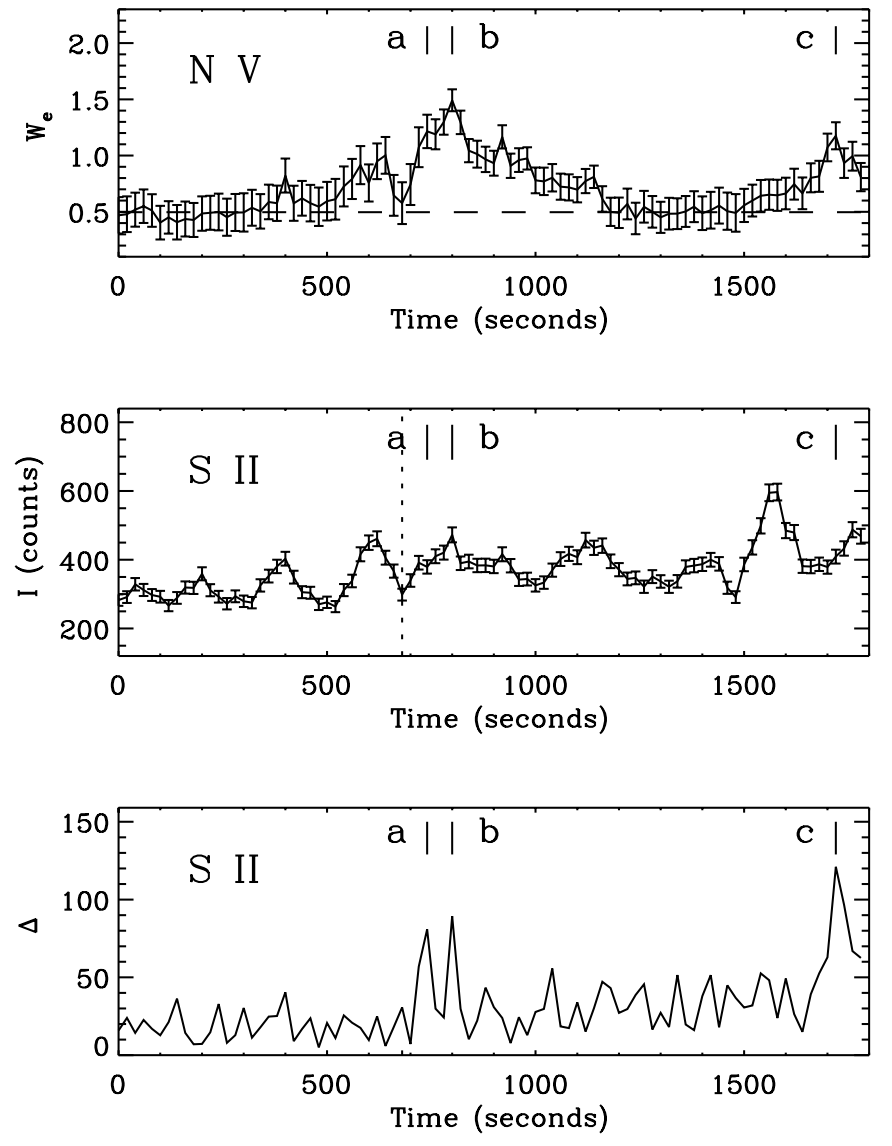

Fig. 3. Top panel: time behaviour along the region marked in Fig. 2 of the $\mathrm{N} v$ wings enhancement factor $W_{\mathrm{e}}$. Horizontal dashed line in the top panel indicate the $W_{\mathrm{e}}$ factor for a $\mathrm{N} v$ profile obtained averaging the first 40 spectra. Mid panel: $\mathrm{S}$ II total intensity (background included). Bottom panel: $\Delta$ quantity as computed throughout Eqs. (2) and (3) (see text). The vertical dotted line indicate the time until which $\mathrm{S}$ II line profiles have been averaged in order to compute the template spectrum $P(\lambda)$ used in Eqs. (2) and (3). Letters mark the point of maximum values of $\Delta$.

panel) the quantity $W_{\mathrm{e}}$ is shown as a function of time. The event around $t=800 \mathrm{~s}$ is evident together with a second (smaller) event around $t=1700 \mathrm{~s}$. Figure 3 (middle panel) shows the total counts in the S II $1253.8 \AA$ line obtained integrating the binned profiles over the spectral interval indicated by vertical dotted lines in the right panel of Fig. 4. An oscillatory pattern with a period around $200 \mathrm{~s}$ can be seen during all the observing period. Oscillatory patterns are a very common feature of chromospheric and transition region lines with coherence scales of the order of several arc-seconds (Doyle et al. 1999). To separate the line intensity fluctuations due to the transition region behaviour from variations in the line shape and/or position that may be associated with the explosive events, we consider the $S$ II $1253.8 \AA$ profile averaged over the first $680 \mathrm{~s}$ (until the dotted vertical line in the mid-panel of Fig. 3) $P(\lambda)$ and calculate, for each $j$ binned profile $O(\lambda)_{j}$, the value $\bar{k}_{j}$ of the constant $k_{j}$ that minimize the expression

$\chi_{j}^{2}=\frac{1}{\left[N_{\mathrm{data}}-1\right]} \sum_{\lambda}\left(\frac{O(\lambda)_{j}-k_{j} P(\lambda)}{\sigma_{\mathrm{data}}(\lambda)}\right)^{2}$. 
N V 1238.8

$\lambda(\AA)$

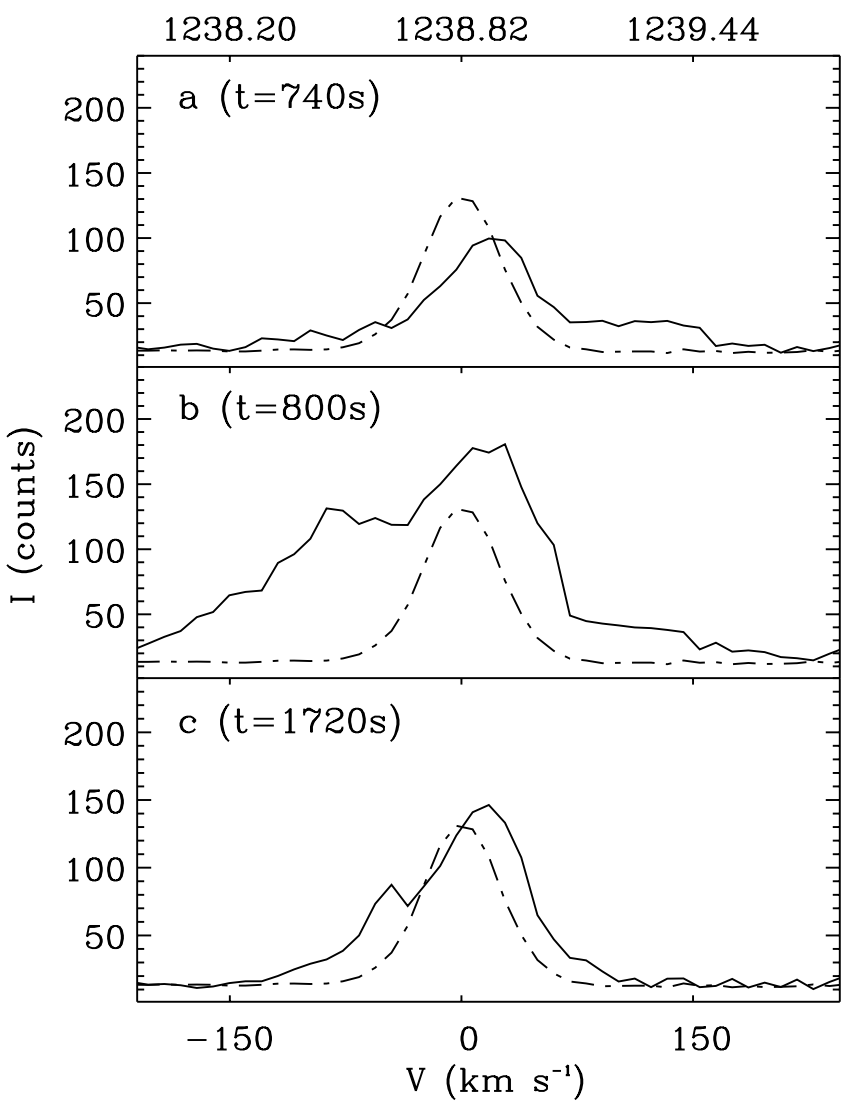

S II 1253.8

$\lambda(\AA)$

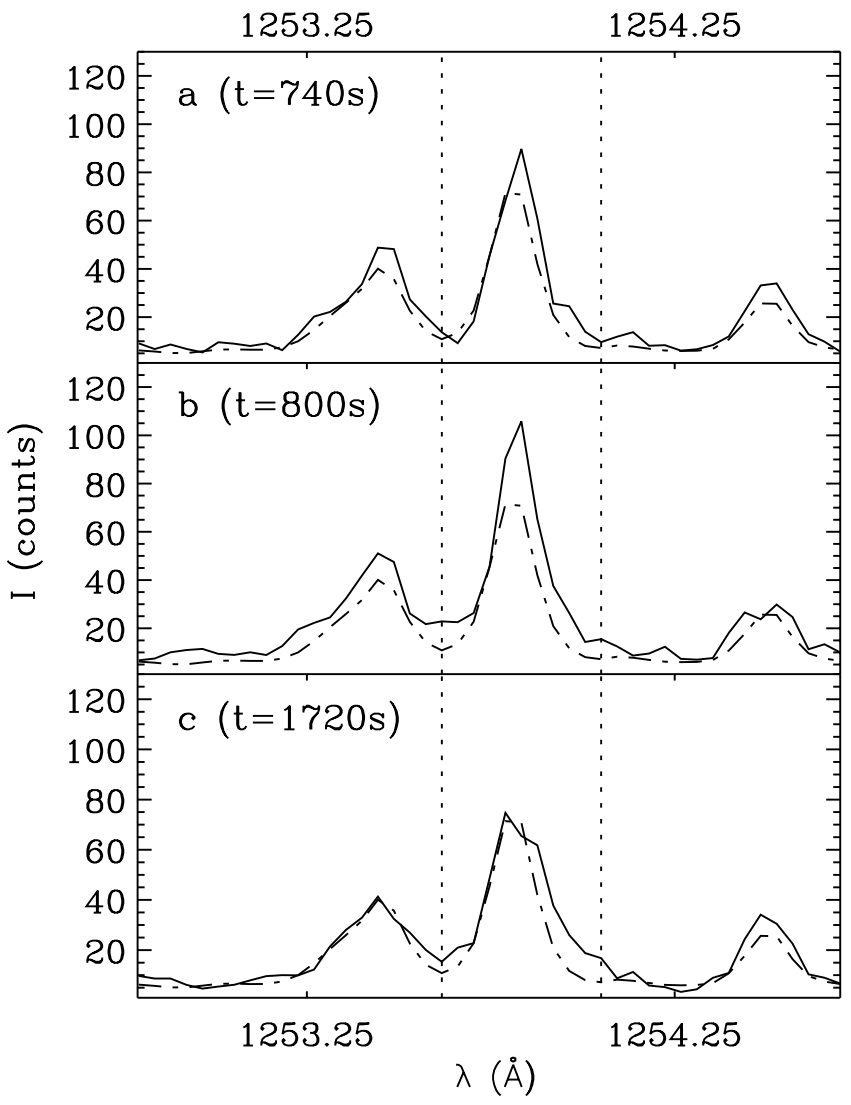

Fig. 4. N v $1238 \AA$ (left panel) and S II $1253 \AA$ (right panel) line profiles during UV explosive events (solid line) together with the line profiles averaged over the first $680 \mathrm{~s}$ (dash dotted line). Letters refer to the positions in time reported in Fig. 3. In the right panel it is also shown, between dotted vertical lines, the spectral interval used in computing Eqs. (2) and (3).

The quantity

$\Delta_{j}=\frac{1}{\left[N_{\text {data }}-1\right]} \sum_{\lambda}\left(O(\lambda)_{j}-\bar{k}_{j} P(\lambda)\right)^{2}$

was, hence, computed for each binned spectral profile and it is shown as a function of time in the bottom panel of Fig. 3. $\Delta$ is a measure of a deviation of the line profile from the average spectrum and characterizes the plasma motions and their velocities. It is evident that the maximum deviation of the line profiles from the average spectrum occur in correspondence with the explosive events seen in $\mathrm{N} v$ at the times marked with the letters a, b and c. In Fig. 4, the N v 1238.8 (left panel) and $\mathrm{S}$ II 1253.8 (right panel) line profiles at the positions marked in Fig. 3 are shown together with the average profiles over the first $680 \mathrm{~s}$. It is not possible to assign one or two particular velocities to the line profiles in Fig. 4 (left panel), instead the plots are best characterised by a distribution of velocities (see also Winebarger et al. 1999) which could indicate a series of energy releases. At position $b$, at the peak of the event, the $\mathrm{N} \mathrm{v}$ line profile shows a strong blue wing witnessing upward velocities larger than $150 \mathrm{~km} \mathrm{~s}^{-1}$. However, in all three time positions the response of the chromospheric and low transition region lines is clearly visible (particularly in event b) although it is quite small. This seems to indicate that intensity enhancements of chromospheric lines of factors 1.6-2 (as reported by Madjarska \& Doyle) could be exceptional and related only to very large events.

\subsection{DTS2 \& DTS3: Coronal behaviour}

The examination of the $\mathrm{N} v 1238.8$ line profiles in these two datasets reveal the presence of two large explosive events. The first explosive event (EE2) was observed on May 242001 around solar $Y=45^{\prime \prime}, \sim 800 \mathrm{~s}$ after the beginning of the observations (see Fig. 5). On October 232001 a second very large event (EE3) was detected around solar $Y=15^{\prime \prime}, \sim 1500 \mathrm{~s}$ after the start of the observations (see Fig. 6). In both cases, binning over 5 pixel wide regions along the slit centered on the explosive event locations was performed (see Figs. 5 and 6 , respectively).

The event (EE2) lasted $\sim 100 \mathrm{~s}$ and was characterized by upward and downward velocities up-to $150 \mathrm{~km} \mathrm{~s}^{-1}$. In the left panel of Fig. 7 the $W_{\mathrm{e}}$ factor (see Eq. (1)) calculated for each binned $\mathrm{N} v$ profile in the selected region is shown. The sharp signature induced by the explosive event is evident. In the same 


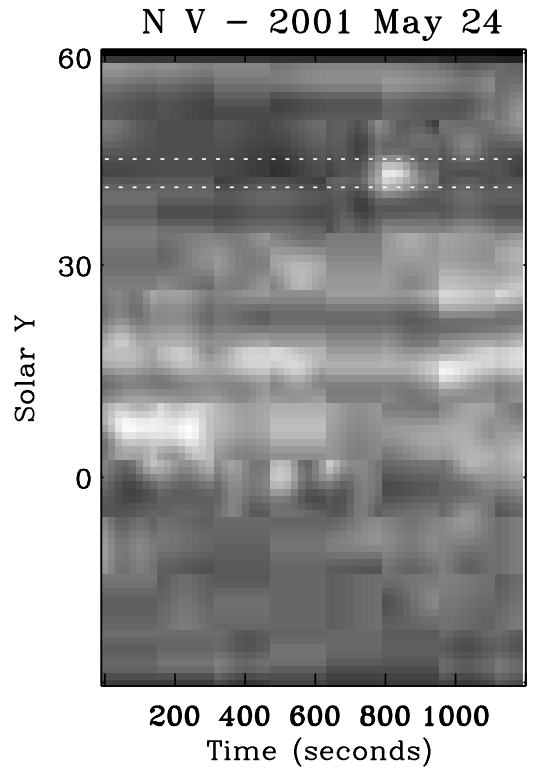

Fig. 5. N v 1238.8 intensity map of the part of the 2001 May 24 dataset here analyzed. The horizontal dotted lines define the portion of the slit along which a large explosive event was observed around $T \sim 800 \mathrm{~s}$.

panel are also shown the contributions to the $W_{\mathrm{e}}$ factor coming from the blue (solid thin line) and the red (dashed thin line) wing $\left(I_{\mathrm{b}} / I_{\text {peak }}\right.$ and $I_{\mathrm{r}} / I_{\text {peak }}$, respectively). In the next two panels of Fig. 7 the line profiles before and during the event are compared. Due to the low counts characterizing the $\mathrm{Mg}$ x line, these profiles were obtained performing a further binning over 4 consecutive spectra in the two intervals marked with vertical dotted lines in the left panel of Fig. 7. In the middle panel of Fig. 7 the $\mathrm{N}$ v 1238.8 profiles before (dashed line) and during (solid line) EE2 are shown. Note the presence of almost symmetrical blue and red enhanced wings together with a central component almost equal to the profile outside the event. In the right panel of Fig. 7 the correspondent $\mathrm{Mg}$ x profiles are shown. It is evident the absence of any detectable difference between the profile before (dashed line) and that during the event (solid line).

The event (EE3) was characterized by a very strong increase in the $\mathrm{N} \mathrm{v}$ integrated intensity (more than a factor of 7) with respect to the values characterizing the same region before the event. It lasted $\sim 400 \mathrm{~s}$ and was characterized by a dominantly blue-shifted emission with velocities up-to $200 \mathrm{~km} \mathrm{~s}^{-1}$. Also in this case we used Eq. (1) to compute the $W_{\mathrm{e}}$ factor, which is reported in the left panel of Fig. 8. From the analysis of the contributions to the $W_{\mathrm{e}}$ factor coming from the blue (solid thin line) and the red (dashed thin line) wing, it is evident that blue-shifted emission is dominant during the entire event. The two portions between the vertical dotted lines represent the intervals over which further binning was computed in order to obtain the $\mathrm{N} v$ (mid panel) and $\mathrm{Mg}$ x (right panel) line profiles $700 \mathrm{~s}$ before (dashed lines) and during the event (solid lines). Also in this case the analysis of the $\mathrm{Mg} \mathrm{x}$ line profiles does not reveal any sign of enhanced wings, instead, there is a strong increase in the first order spectrum. The $\mathrm{Mg}$ x line intensity was obtained using a multi-Gaussian fitting technique; its temporal

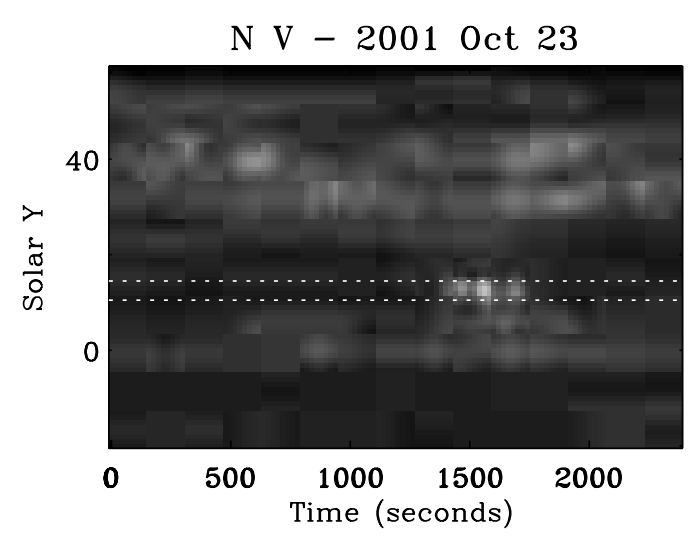

Fig. 6. N v 1238.8 intensity map of the part of the 2001 October 23 dataset here analyzed. The horizontal dotted lines define the portion of the slit along which a very large explosive event was observed around $T \sim 1600 \mathrm{~s}$.

behaviour (see Fig. 9 top panel) does not show any significant variation during the explosive event while an increase of a factor $\sim 3$ is observed in the case of $S_{\text {II }} 1250.58 \AA$ line (see Fig. 9 bottom panel). In these conditions the first order blends within $\mathrm{Mg} \mathrm{x}$ are likely to account for $\sim 5-6 \%$ of the $\mathrm{Mg}$ x total counts, explaining the small increase in the $\mathrm{Mg} \mathrm{x}$ intensity at the peak of the event (see Fig. 9 top panel). Thus even during this very large transition region event no coronal response is observed.

\section{Discussion}

Classically, the transition region is considered as a thin thermal interface between the cool chromosphere $\left(T \sim 10^{4} \mathrm{~K}\right)$ and the hotter corona $\left(T \sim 10^{6} \mathrm{~K}\right)$ (e.g. Moore \& Fung 1972; Gabriel 1976; Athay 1981). In these conditions the upper transition region $\left(T>2.5 \times 10^{5} \mathrm{~K}\right)$ is mainly heated by thermal conduction from the corona where the heating is assumed to occur. The failure of these models in reproducing the transition region emission at temperatures below $10^{5} \mathrm{~K}$ lead some authors to hypothesize the presence of a mixture of cold $\left(T<10^{5} \mathrm{~K}\right)$ low-lying small $\left(<10^{4} \mathrm{~km}\right)$ loops prevalently located in the network and accounting for the emission seen in lines formed at $T<10^{5}$ K (Antiochos \& Noci 1986; Dowdy et al. 1986). In particular, Dowdy et al. (1986) proposed the coexistence with hot coronal funnels accounting for the upper transition region emission. In this model the bulk of the lower transition region emission could come from structures disconnected from the corona.

Doschek et al. (1976) and Mariska et al. (1978) find that the limb emission of lines formed above $10^{5} \mathrm{~K}$ peaks 4 arcsecs above the white-light limb and two arcsecs $(\sim 1400 \mathrm{~km})$ above the point where the peak of the emission of lines formed around $5 \times 10^{4} \mathrm{~K}$ falls. In classical loop models bridging from the chromosphere to the corona these two temperatures are only separated in height by $\sim 100 \mathrm{~km}$ (see Mariska 1992). Based on this and others observational facts, Feldman (1983, 1987) and Feldman \& Laming (1994) suggested that most of the plasma at temperatures below $5 \times 10^{5} \mathrm{~K}$ resides in unresolved fine, thermally insulated, structures (UFS) that do not reach coronal temperatures. In a more recent work, 

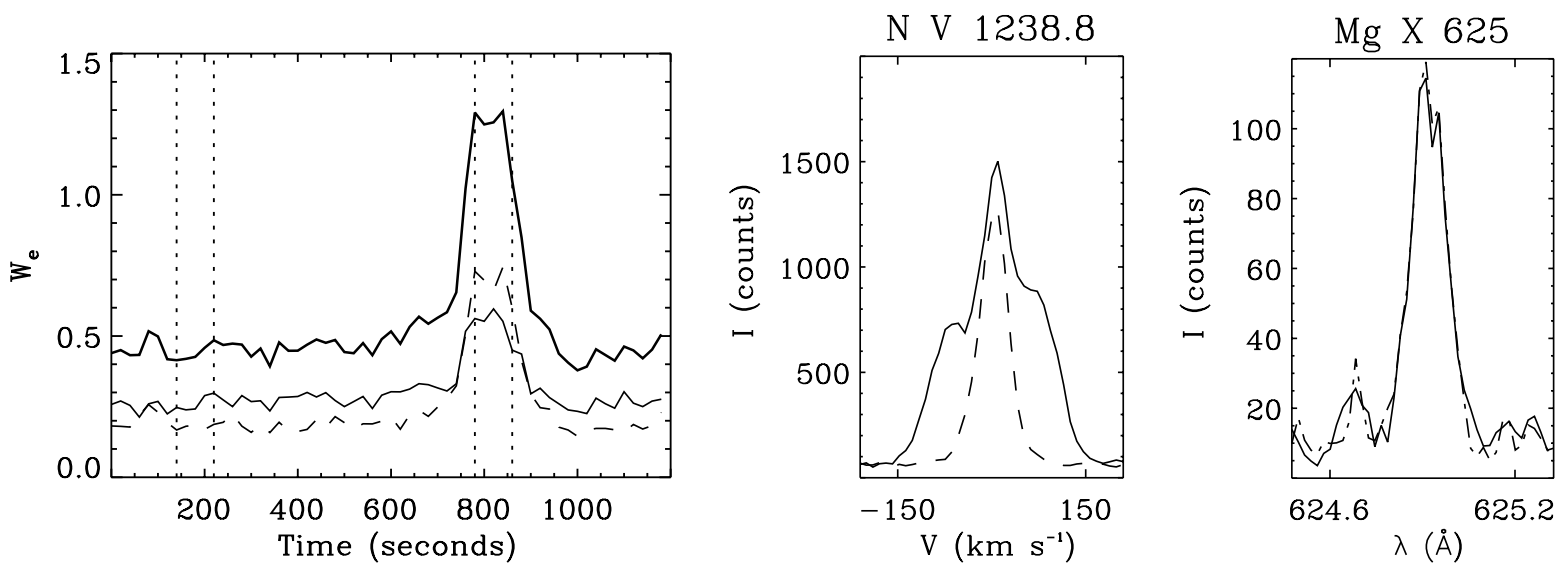

Fig. 7. Time behaviour along the region marked in Fig. 5 of the $\mathrm{N} v$ wings enhancement factor $W_{\mathrm{e}}$ showing a strong explosive event (EE2) lasting $\sim 100 \mathrm{~s}$ (left panel). In the same panel are also shown the contributions to the $W_{\mathrm{e}}$ factor coming from the blue (solid thin line) and the red (dashed thin line) wing $\left(I_{\mathrm{b}} / I_{\text {peak }}\right.$ and $I_{\mathrm{r}} / I_{\text {peak }}$, respectively). The $\mathrm{N} \mathrm{v} 1238 \AA$ and $\mathrm{Mg}$ x $625 \AA$ line profiles before (around $\left.t=180 \mathrm{~s}\right)$ and during the explosive event are shown in the central and right panels using dashed and solid lines, respectively. The profiles were obtained with a further binning over the time intervals marked with vertical dotted lines in the left panel.
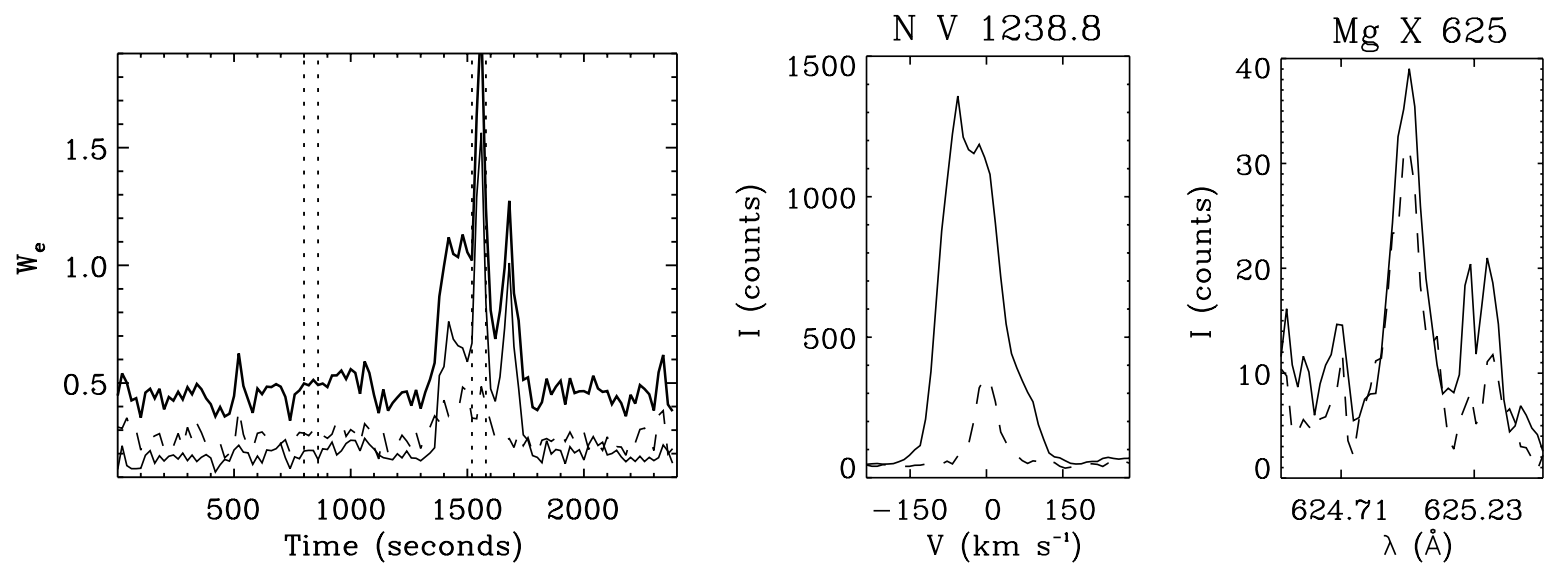

Fig. 8. Time behaviour along the region marked in Fig. 6 of the $\mathrm{N} v$ wings enhancement factor $W_{\mathrm{e}}$ showing a strong explosive event lasting $\sim 400 \mathrm{~s}$ (left panel, solid thick line). In the same panel are also shown the contributions to the $W_{\mathrm{e}}$ factor coming from the blue (solid thin line) and the red (dashed thin line) wing $\left(I_{\mathrm{b}} / I_{\text {peak }}\right.$ and $I_{\mathrm{r}} / I_{\text {peak }}$, respectively). The $\mathrm{N} \mathrm{v} 1238 \AA$ and $\mathrm{Mg} \mathrm{x} 625 \AA$ line profiles before (around $t=820 \mathrm{~s}$ ) and during the explosive event are shown in the central and right panels using dashed and solid lines, respectively. The profiles were obtained with a further binning over the time intervals marked with vertical dotted lines in the left panel. The small increase in the $\mathrm{Mg} \mathrm{x}$ increase is due to first order blends (see text for details).

Feldman et al. (1999) moved the separation temperature up to $8 \times 10^{5} \mathrm{~K}$.

Wikstøl et al. (1998) have critically evaluated the arguments leading to the UFS hypothesis. They showed that dynamic classical transition region models can account for several (if not all) of the observational evidence without the necessity of introducing the UFS. More recently, small scale looplike structures extending inside the super-granulation cells, has been found particularly at the boundaries of the supergranulation network (Warren \& Winebarger 2000). Many of these structures are characterized by width equal to the spatial resolution and length from a few to $\sim 30$ arcsec (Warren \& Winebarger 2000; Landi et al. 2000; Feldman et al. 1999).

At transition region densities and temperatures the magnetic pressure dominates over the plasma pressure and all plasma flows are forced to follow the magnetic field lines. Our $\mathrm{Mg}$ x results show that, at sites of strong accelerations, the plasma is not heated to coronal temperatures indicating that energy is mostly used to accelerate it. The presence of velocities up to $250 \mathrm{~km} \mathrm{~s}^{-1}$ would, within this framework, suggest a possible role for kinetic and enthalpy energy fluxes associated with explosive events in heating the quiet Sun corona. There have been several attempts at estimating the kinetic energy (e.g. Dere et al. 1989; Porter \& Dere 1991; Dere 1994; Winebarger et al. 1999, 2002a). The most recent of these (Winebarger et al. 2002a) considered the velocity distribution function of the emitting plasma deriving upward directed energy fluxes of $4 \times 10^{4} \mathrm{erg} \mathrm{cm}^{-2} \mathrm{~s}^{-1}$ where kinetic energy fluxes represented only $\sim 5 \%$ of the total (mostly enthalpy fluxes). These values would indicate that explosive events can account for $\sim 10 \%$ of the coronal energy requirement. This, combined with our results, would therefore suggest that UV explosive events do not provide a major coronal contribution. 


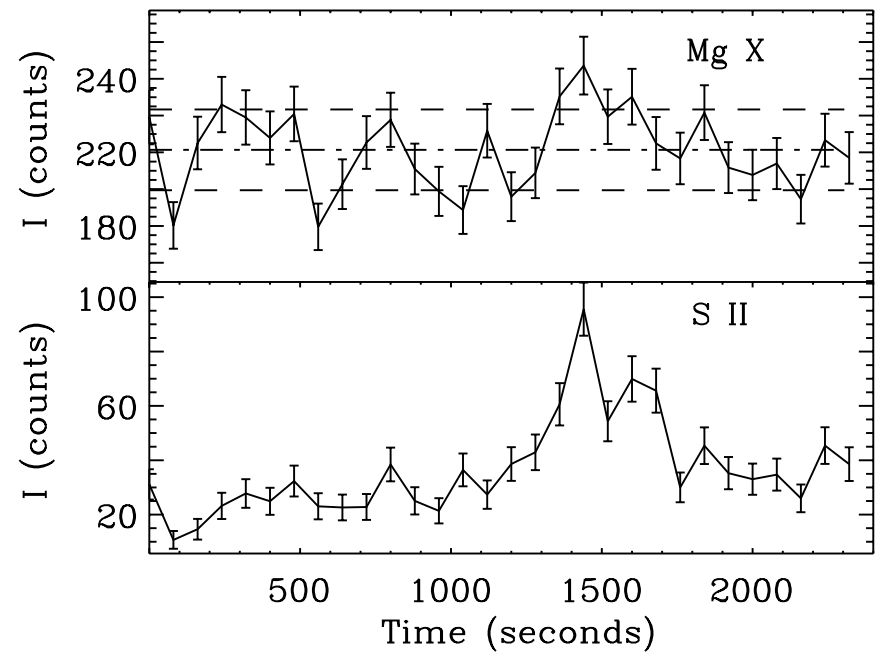

Fig. 9. $\mathrm{Mg}$ x $625 \AA$ (top) and S II 1250.58 (bottom) line intensities along the region marked in Fig. 6. The horizontal dash-dotted line represents the average intensity value, while the $1 \sigma$ levels of the $\mathrm{Mg} \mathrm{x}$ intensity values displayed are shown with dashed lines.

It should be also noted that, as the plasma travels at velocities higher than the local sound speed $\left(\sim 70 \mathrm{~km} \mathrm{~s}^{-1}\right.$ at $T=1.8 \times 10^{5} \mathrm{~K}$ ) it is likely to form a traveling shock that would compress and heat the plasma at locations not coincident with the site where maximum acceleration is observed in transition region lines. If, for instance, explosive events occur in small loops $\left(\sim 10^{\prime \prime}\right)$ it could be possible that brightening at higher temperatures could occur at locations not covered by the SUMER slit as suggested by Innes (2001). Recent work by Winebarger et al. (2002b) indicates that significant fluctuations in the TRACE 171 wave-band is observed in $35 \%$ of the pixels which show enhanced wings in transition region lines over an area within an active region. This is consistent with work by Erdélyi et al. (1999) who observed an explosive event in $\mathrm{N} v$ which did not show any evidence of activity in $\mathrm{Mg} \mathrm{X}$, yet the TRACE 171 wave-band clearly showed enhanced activity. The line formation temperature of Fe Ix and Fe $\mathrm{x}$, which are present in the TRACE 171 wave-band, are $6.3 \times 10^{5}$ and $1 \times 10^{6} \mathrm{~K}$ based on the Arnaud \& Raymond (1992) ionization balance calculations. These temperatures are approximately a factor of two less than some other calculations and since they seem to fit the TRACE data better would perhaps suggest a reevaluation of the $\mathrm{Fe}$ atomic data is required. However, it should also be pointed out that there is an unknown contribution from the O v $172 \AA$ and O vi $173 \AA$ lines in the TRACE 171 waveband, that could be particularly relevant during transient events. Explosive events have spatial scales $(\sim 2000 \mathrm{~km})$ much larger than the thickness of the transition region in classical loop models. The absence of any observable variation in the plasma at $10^{6} \mathrm{~K}$ temperatures, coupled with the above formation temperature of Fe IX, would be consistent with the idea of a transition region dominated by structures not connected with the corona as suggested by Feldman (1983) and Feldman et al. (1999).

Our results also help in shedding some light on where in the solar atmosphere the magnetic reconnection powering explosive events occur. Several possibilities are present in the literature. Dere et al. (1991) and Innes et al. (1997a) suggest that Petschek reconnection (Petschek 1964) occurs directly in plasma at transition region temperatures leading to the creation of bi-direction jets. From the analysis of centimeter radio emission and ultraviolet coronal lines Benz \& Krucker (1999) proposed that reconnection occur high in the corona and generate high energy particles beams that heat and accelerate the chromospheric plasma leading to the transition region signature. Emission in coronal lines follows due to evaporating plasma expanding into the corona and cooling by conducting part of the energy thereby increasing the emission of the $1 \mathrm{MK}$ plasma a few minutes later that the peak in the plasma to transition region temperatures. More recently, Ryutova et al. (2001), Tarbell et al. (2000) and Ryutova \& Tarbell (2000) proposed that reconnection in photosphere leads to shocks that accelerate the plasma at transition region temperatures. The absence of variations in the $\mathrm{Mg} \mathrm{x}$ emissions during and after the explosive events observed here (see Fig. 9) would exclude the coronal origin of these events while the presence of enhanced emission in chromospheric lines would favour a location in the chromosphere. In this context our results add further constrains to future works on explosive event modeling. Recently, Roussev et al. (2001) investigated the response of various 2D environments approximating the "quiet" solar atmosphere to a magnetic reconnection event. Although these particular calculations went much further than previous modeling, e.g. they explored the MHD output in terms of synthesized resonance transitions of Li-like ions, involving non-equilibrium ionization, the resulting output was still far from being comparable to observations.

Acknowledgements. Research at Armagh Observatory is grantaided by the N. Ireland Dept. of Culture, Arts and Leisure. The SUMER project is financially supported by DLR, CNES, NASA, and PRODEX. This work was supported by PPARC grants PPA/GIS/1999/00055 and PPA/V/S/1999/00628. We are also grateful to P. Lemaire for his help with the SUMER observations, obtained during MEDOC Campaign 8, and to K. Dere for his help with HRTS spectra.

\section{References}

Antiochos, S. K., \& Noci, G. 1986, ApJ, 301, 440

Arnaud, M., \& Raymond, J. C. 1992, ApJ, 398, 39

Athay, R. G. 1981, ApJ, 249, 340

Banerjee, D., O'Shea, E., Doyle, J. G., \& Goosens, M. 2001, A\&A, 371,1137

Benz, A. O., \& Krucker, S. 1999, A\&A, 341, 286

Brković, A., Rüedi, L., Solanki, S. K., et al. 2000, A\&A, 353, 1083

Brueckner, G. E., Patterson, N. P., \& Scherrer, V. E. 1976, Sol. Phys., 47, 127

Brueckner, G. E., \& Bartoe, J.-D. F. 1983, ApJ, 272, 329

Chae, J., Wang, H., Lee, C. Y., Goode, P. R., \& Schühle, U. 1998a, ApJ, 497, L109

Chae, J., Wang, H., Lee, C. Y., Goode, P. R., \& Schühle, U. 1998b, ApJ, 504, L123

Cheng, C. C., \& Kjeldseth-Moe, O. 1990, in Dynamics of Solar Flares, ed. B. Schmieder, \& E. Priest, Observatoire de Paris, DASOP, 101

Cohen, L., Feldman, U., \& Doschek, G. A. 1978, ApJS, 37, 393

Curdt, W., Brekke, P., Feldman, U., et al. 2001, A\&A, 375, 591 
Dammasch, I. E., Hassler, D. M., Wilhelm, K., \& Curdt, W. 1999, ESA SP-446, 263

Dere, K. P., Bartoe, J.-D. F., \& Brueckner, G. E. 1984, ApJ, 281, 870

Dere, K. P., Bartoe, J.-D. F., Brueckner, G. E., et al. 1987, Sol. Phys., 114,223

Dere, K. P., Bartoe, J.-D. F., \& Brueckner, G. E. 1989, Sol. Phys., 123,41

Dere, K. P., Bartoe, J.-D., Brueckner, et al. 1991, J. Geophys. Res., 96, 9399

Dere, K. P. 1992, in Solar Wind Seven, ed. E. Marsch, \& R. Schwenn, 11

Dere, K. P. 1994, Adv. Space Res., 14(4), 13

Doschek, G. A., Feldman, U., Vanhoosier, M. E., \& Bartoe, J.-D. F. 1976, ApJS, 31, 417

Dowdy, J. F. Jr., Rabin, D., \& Moore, R. L. 1986, Sol. Phys., 105, 35

Dowdy, J. F. Jr. 1993, ApJ, 411, 406

Doyle, J. G., van den Oord, G. H. J., O’Shea, E., \& Banerjee, D. 1998, Sol. Phys., 181, 51

Doyle, J. G., van den Oord, G. H. J., O'Shea, E., \& Banerjee, D. 1999, A\&A, 347, 335

Erdélyi, R., De Pontieu, B., \& Sarro, L. M. 1999, in Magnetic Fields and solar Processes, Florence, Italy, ESA SP-448, 1345

Feldman, U., Doschek, G. A., \& Mariska, J. T. 1979, ApJ, 229, 369

Feldman, U. 1983, ApJ, 275, 367

Feldman, U. 1987, ApJ, 320, 426

Feldman, U., \& Laming, J. M. 1994, ApJ, 434, 370

Feldman, U., Widing, K. G., \& Warren, H. P. 1999, ApJ, 522, 1133

Gabriel, A. H. 1976, Phil. Trans. Roy. Soc. Lond., A 281, 339

Gallagher, P. T., Phillips, K. J. H., Harra-Murnion, L. K., et al. 1999, A\&A, 348, 251

Harrison, R. A. 1997, Sol. Phys., 175, 467

Harrison, R. A., Lang, J., Brooks, D. H., \& Innes, D. E. 1999, A\&A, 351,1115

Innes, D. E., Brekke, P., Germerott, D., \& Wilhelm, K. 1997a, Sol. Phys., 175, 341

Innes, D. E., Inhester, B., Axford, W. I., \& Wilhelm, K. 1997b, Nature, 386,811

Innes, D. E. 2001, A\&A, 378, 1067

Kjeldseth-Moe, O., \& Cheng, C. C. 1994, Space Sci. Rev., 70, 85

Koutchmy, S., Harra, H., Suematsu, Y., \& Reardon, K. 1997, A\&A, 320,33
Krucker, S., Benz, A. O., Bastian, T. S., \& Acton, L. W. 1997, ApJ, 488, 499

O'Shea, E., Banerjee, D., Doyle, J. G., Fleck, B., \& Murtagh, F. 2001, A\&A, 368, 1095

Landi, E., \& Landini, M. 1998, A\&AS, 133, 411

Landi, E., Mason, H. E., Lemaire, P., \& Landini, M. 2000, A\&A, 357, 743

Lemaire, P., Wilhelm, K., Curdt, W., et al. 1997, Sol. Phys., 170, 105

Madjarska, M. S., \& Doyle, J. G. 2002, A\&A, 382, 319

Mariska, J. T., Feldman, U., \& Doschek, G. A. 1978, ApJ, 226, 698

Mariska, J. T. 1992, The Solar Transition Region (University Press, Cambridge)

Moore, R. L., \& Fung, P. C. W. 1972, Sol. Phys., 23, 78

Moses, D., \& Cook, J. W. 1994, Space Sci. Rev., 70, 81

Pérez, M. E., Doyle, J. G., Erdélyi, R., \& Sarro, L. M. 1999, A\&A, 342,279

Petschek, H. E. 1964, in AAS-NASA Symp. on the Physics of Solar Flares, ed. W. N. Hess, NASA-SP, 50, 425

Porter, J. G., \& Dere, K. P. 1991, ApJ, 370, 775

Roussev, I., Doyle, J. G., Galsgaard, K., \& Erdélyi, R. 2001c, A\&A, 380,719

Ryutova, M. P., \& Tarbell, T. D. 2000, ApJ, 541, L29

Ryutova, M. P., Habbal, S. R., Woo, \& Tarbell, T. D. 2001, Sol. Phys., 200, 213

Sandlin, G. D., Bartoe J.-D. F., Brueckner G. E., et al. 1986, ApJS, 61, 801

Siegmund, O. H., Stock, J. M., Marsh, D. R., et al. 1994, Proc. SPIE, 2280, 89

Tarbell, T. D., Ryutova, M. P., \& Shine, R. 2000, Sol. Phys., 193, 195

Teriaca, L., Madjarska, M. S., \& Doyle, J. G. 2001, Sol. Phys., 200, 91

Winebarger, A. R., Emslie, A. G., Mariska, J. T., \& Warren, H. P. 1999, ApJ, 526, 471

Winebarger, A. R., Emslie, A. G., Mariska, J. T., \& Warren, H. P. 2002, ApJ, 565, 1298

Winebarger, A. R., Updike, A. C. \& Reeves, K. K. 2002b, ApJ, submitted

Wikstøl, Ø., Judge, P. G., \& Hansteen, V. 1998, ApJ, 501, 895

Wilhelm, K., Curdt, W., Marsch, et al. 1995, Sol. Phys., 162, 189

Wilhelm, K., Lemaire, P., Curdt, W., et al. 1997, Sol. Phys., 170, 75

Wilhelm, K., Innes, D. E., Curdt, W., et al. 1998, in Solar Jets and Coronal Plumes, Guadalupe, France, ESA-SP 421, 103 